\title{
ACOUSTIC WAVES IN LONG RANGE RANDOM MEDIA*
}

\author{
RENAUD MARTY ${ }^{\dagger}$ AND KNUT SOLNA ${ }^{\ddagger}$
}

\begin{abstract}
We consider waves propagating through multiscale media. Much is known about waves propagating through a medium that satisfies a scale separation assumption with random fluctuations on a microscale. Here we go beyond this situation and consider waves propagating through a medium defined in terms of a long range process. Such a medium can, for instance, be modeled in terms of a one-dimensional fractional Brownian motion with variations on a continuum of scales. Fractal medium models are used to model, for example, the heterogeneous earth and the turbulent atmosphere. We set forth a framework using the theory of rough paths in which propagation problems of this nature can be analyzed in the case with anticipative medium fluctuations with a Hurst exponent $H>1 / 2$. We show how the wave interacts with the medium fluctuations in this case and that the interaction is qualitatively different from the situation where the medium satisfies a separation of scales assumption. In the long range case considered here the travel time depends strongly on the particular medium realization, but in fact the pulse shape does not.
\end{abstract}

Key words. wave propagation, random media, long range processes, fractional Brownian motion

AMS subject classifications. 34F05, 34E10, 37H10, 60H20

DOI. $10.1137 / 07068610 \mathrm{X}$

1. Introduction. Modeling in terms of a multiscale medium is important for propagation problems in, for instance, the earth's crust, the turbulent atmosphere, turbulent boundary layers, sea ice, and outer space $[9,15,19,20,34,42,44]$. Communication, remote sensing, and laser beam propagation schemes are affected by bad weather and multiscale medium variations. Large scale research projects (the ABLE ACE program Kirtland AFB, for instance [43]) have focused on gathering atmospheric turbulence data and numerically simulating propagation of wave fields through synthetic turbulence models that derive from these. Above a boundary layer atmospheric turbulence may occur within a stratified environment, and the turbulent temperature variations may be highly anisotropic; see $[16,36]$. Rough and long range medium fluctuations associated with multiscale modeling are also important in medical imaging, device modeling, and nuclear technology, to name a few. The zone in between different tissue types (or in between different dielectrica) may in particular be strongly heterogeneous with variation on a continuum of scales. In general, the detailed pointwise variation of a multiscale medium, the refractive index, say, cannot be identified. However, the statistics of this variation can be characterized. Optimal design of, for instance, imaging and communication algorithms requires insight about how the wave is affected by the rough medium fluctuations, that is, the nonlinear coupling between medium and wave field statistics. This is particularly the case with modern high resolution sampling and imaging technology. Insight about the wave medium interaction is also important in a range of other applications like design of sound-absorbing materials and nondestructive evaluation of fractured materials. A good understanding

*Received by the editors March 22, 2007; accepted for publication (in revised form) September 12, 2008; published electronically January 28, 2009.

http://www.siam.org/journals/siap/69-4/68610.html

†Institut Elie Cartan Nancy, Nancy-Université, CNRS, INRIA, Boulevard des Aiguillettes, B.P. 239, F-54506 Vandœuvre lès Nancy, France (renaud.marty@iecn.u-nancy.fr).

$\ddagger$ Department of Mathematics, University of California at Irvine, Irvine, CA 92697 (ksolna@math. uci.edu). Work partially supported by DARPA grant N00014-02-1-0603, ONR grant N00014-02-10090, NSF grant 0307011, and the Sloan Foundation.

1065 
of how the wave interacts with variations on a continuum of small length scales is therefore important.

Propagation of high frequency waves in smooth media is well understood. A lot is also known about propagation in heterogeneous media that vary on a well-defined microscale. However, propagation in rough or multiscale media is not so well understood. We will look at how propagating pulses interact with rough variations in the medium. In $[10,11,12]$ we considered propagation in rough media when the wave phenomenon was modeled in terms of the paraxial or forward approximation. In [37] we considered the full wave equation and a discrete multiscale medium. Here, we continue this line of research by analyzing the full wave equation in the context of a one-dimensional continuous multiscale medium modeled in terms of a long range process with slowly decaying correlations, and we consider in particular fractional Brownian motion-based media.

In the homogenization or effective medium regime, with the width of the propagating pulse being large compared to the scale of the medium fluctuations and propagation distances on the scale of the wavelength, the rapidly varying properties of the medium can be replaced by their homogenized or averaged values. However, over long propagation distances the accumulated effect of the scattering, associated with the medium microstructure, gradually changes the pulse beyond the geometrical effects of the high frequency analysis in the smooth homogenized medium. These modifications depend in general on the particular medium realization. Thus, to describe the propagation phenomenon it is not enough to consider only the mean wave field; one should also aim at describing the character of the fluctuations in the wave field. A mathematical theory for pulse propagation has been developed in $[1,6,14,25]$. It deals with pulses in a particular realization of the random medium, and it explains why in many cases the evolution of the pulse shape is to leading order deterministic. We refer to this phenomenon as pulse stabilization. So far, two salient features of this "pulse shaping" theory have been that it assumes a one-dimensional medium and a separation of scales for the medium heterogeneities, that is, that the medium has features on microscales which are well separated from the macroscale. However, as explained above, many empirical studies suggest that, for instance, the earth's crust should be modeled as containing fluctuations on a continuum of length scales. Stabilization and pulse shaping in a two scale medium with slow lateral variations in the medium has been analyzed in [38]. Here, we generalize the pulse shaping theory for a two scale medium to the long range multiscale case.

We analyze acoustic waves propagating in a one-dimensional medium, modeled in terms of a long range process. As a particular example we consider media defined in terms of fractional Brownian motion. Fractional Brownian motion is a Gaussian (self-similar) stochastic process and is often used as a model for processes containing fluctuations on a continuum of length scales, for instance for modeling of turbulent environments. The Hurst exponent $H$ characterizes the roughness of the fractional Brownian motion, and the value $H=1 / 2$ gives standard Brownian motion. In the simplest case with $H=1 / 2$ the medium model that we consider satisfies a separation of scales assumption. For $H \neq 1 / 2$ the medium contains long range interactions and variations on many scales. In this case the correlations in the medium have only polynomial decay, and our objective is to analyze the effects such long range correlations have on the propagating wave. Here, we shall analyze the case with $H>1 / 2$ corresponding to persistent fluctuations so that consecutive increments of the process are positively correlated [13]. In fact, we shall show that the pulse shape is not affected by the random medium fluctuations to leading order. However, the travel time of the 
pulse depends on the particular medium realization, and the travel time fluctuations are large relative to the period of the pulse.

A number of studies of wave interaction with a fractal or multiscale object deal with scattering caused by fractal interfaces. However, some authors have explored wave-interaction with deterministic fractal media using numerical simulations [5, 21, 39]. Here, we present a mathematical analysis of acoustic pulse transmission through a random fractal and illustrate our theoretical results with numerical simulations. In section 2 we introduce the problem and review the basic wave decomposition approach and the classical scale separation result. Next, in section 3 we introduce the multiscale medium and summarize how the pulse shaping theory generalizes to these media. In section 4, we illustrate our theoretical results with numerical simulations. Finally, section 5 is devoted to the derivation of the main result.

2. Wave decomposition. The governing equations are the Euler equations giving conservation of moments and mass:

$$
\begin{gathered}
\rho(z) \frac{\partial u}{\partial t}(z, t)+\frac{\partial p}{\partial z}(z, t)=0 \\
\frac{1}{K(z)} \frac{\partial p}{\partial t}(z, t)+\frac{\partial u}{\partial z}(z, t)=0
\end{gathered}
$$

where $t$ is the time, $z$ is the depth into the medium, $p$ is the pressure, and $u$ the particle velocity. The medium parameters are the density $\rho$ and the bulk-modulus $K$ (reciprocal of the compressibility). We assume that $\rho$ is a constant identically equal to one in our nondimensionalized units and that $1 / K$ is modeled as follows:

$$
\frac{1}{K(z)}= \begin{cases}1+\varepsilon^{\kappa} \nu\left(\frac{z}{\varepsilon^{2}}\right) & \text { for } z \in[0, Z], \\ 1 & \text { for } z \in \mathbb{R}-[0, Z],\end{cases}
$$

where $\kappa \geq 0$. We introduce the right- and left-going waves

$$
A=p+u \quad \text { and } \quad B=u-p,
$$

where the boundary conditions are of the form

$$
A(z=0, t)=f\left(t / \varepsilon^{\tau}\right) \text { and } B(z=Z, t)=0,
$$

for a positive real number $\tau>0$ and a source function $f$. In order to deduce a description of the transmitted pulse, we open a window of size $\varepsilon^{\tau}$ in the neighborhood of the travel time of the homogenized medium and define the processes

$$
a^{\varepsilon}(z, s)=A\left(z, z+\varepsilon^{\tau} s\right) \quad \text { and } \quad b^{\varepsilon}(z, s)=B\left(z,-z+\varepsilon^{\tau} s\right) .
$$

Observe that the background or homogenized medium in our scaling has a constant speed of sound equal to unity and that the medium is matched so that in the frame introduced in (2.6) the pulse-shapes of the right- and left-going waves are constant in the slab if $\nu \equiv 0$ or if we consider the homogenized medium [14]. We introduce next the Fourier transforms $\widehat{a}^{\varepsilon}$ and $\widehat{b}^{\varepsilon}$ of $a^{\varepsilon}$ and $b^{\varepsilon}$, respectively,

$$
\widehat{a}^{\varepsilon}(z, \omega)=\int e^{i \omega s} a^{\varepsilon}(z, s) d s \quad \text { and } \quad \widehat{b}^{\varepsilon}(z, \omega)=\int e^{i \omega s} b^{\varepsilon}(z, s) d s,
$$


that satisfy

$$
\begin{array}{ll}
\frac{d \widehat{a}^{\varepsilon}}{d z}=\frac{i \omega}{2 \varepsilon^{\tau-\kappa}} \nu\left(\frac{z}{\varepsilon^{2}}\right)\left(\widehat{a}^{\varepsilon}-e^{-2 i \omega z / \varepsilon^{\tau}} \widehat{b}^{\varepsilon}\right), & \widehat{a}^{\varepsilon}(0, \omega)=\widehat{f}(\omega), \\
\frac{d \widehat{b}^{\varepsilon}}{d z}=\frac{i \omega}{2 \varepsilon^{\tau-\kappa}} \nu\left(\frac{z}{\varepsilon^{2}}\right)\left(e^{2 i \omega z / \varepsilon^{\tau}} \widehat{a}^{\varepsilon}-\widehat{b}^{\varepsilon}\right), & \widehat{b}^{\varepsilon}(Z, \omega)=0 .
\end{array}
$$

Following $[6,14]$, we express the previous system of equations in term of propagator $P_{\omega}^{\varepsilon}(z)$, which can be written as

$$
P_{\omega}^{\varepsilon}(z)=\left(\begin{array}{cc}
\alpha_{\omega}^{\varepsilon}(z) & \overline{\beta_{\omega}^{\varepsilon}}(z) \\
\beta_{\omega}^{\varepsilon}(z) & \overline{\alpha_{\omega}^{\varepsilon}}(z)
\end{array}\right)
$$

and which satisfies

$$
\frac{d P_{\omega}^{\varepsilon}}{d z}(z)=\frac{1}{\varepsilon^{\gamma}} H_{\omega}\left(\frac{z}{\varepsilon^{\tau}}, \frac{z}{\varepsilon^{2}}\right) P_{\omega}^{\varepsilon}(z), \quad P_{\omega}^{\varepsilon}(z=0)=\left(\begin{array}{cc}
1 & 0 \\
0 & 1
\end{array}\right),
$$

with $\gamma=\tau-\kappa$ and

$$
H_{\omega}\left(z_{1}, z_{2}\right)=\frac{i \omega}{2} \nu\left(z_{2}\right)\left(\begin{array}{cc}
1 & -e^{-2 i \omega z_{1}} \\
e^{2 i \omega z_{1}} & -1
\end{array}\right) .
$$

Defining next the transmission coefficient $T_{\omega}^{\varepsilon}$ and the reflection coefficient $R_{\omega}^{\varepsilon}$ by

$$
T_{\omega}^{\varepsilon}(z)=\frac{1}{\overline{\alpha_{\omega}^{\varepsilon}(z)}} \quad \text { and } \quad R_{\omega}^{\varepsilon}(z)=\frac{\beta_{\omega}^{\varepsilon}(z)}{\overline{\alpha_{\omega}^{\varepsilon}(z)}}
$$

we can write

$$
a^{\varepsilon}(Z, s)=\frac{1}{2 \pi} \int e^{-i s \omega} T_{\omega}^{\varepsilon}(Z) \widehat{f}(\omega) d \omega
$$

and

$$
b^{\varepsilon}(0, s)=\frac{1}{2 \pi} \int e^{-i s \omega} R_{\omega}^{\varepsilon}(Z) \widehat{f}(\omega) d \omega .
$$

Henceforth, we shall study the asymptotics of the propagator $P_{\omega}^{\varepsilon}$ in order to characterize $a^{\varepsilon}$ and $b^{\varepsilon}$ as $\varepsilon$ goes to 0 .

We recall now what happens in a "short range" model when $\tau=1$ and $\kappa=0$. We assume that $\nu$ is a centered Markov process with an invariant probability measure whose generator satisfies the Fredholm alternative. It is well known $[6,14]$ that under these assumptions, in order to characterize the transmitted pulse, the propagator equations $P_{\omega}^{\varepsilon}$ can be replaced by an effective system of stochastic differential equations from which we can deduce that, as $\varepsilon$ goes to 0 ,

$$
a^{\varepsilon}(Z, s) \longrightarrow \widetilde{a}(Z, s),
$$

with

$$
\widetilde{a}(Z, s)=(f * G)(s-B),
$$

Copyright $@$ by SIAM. Unauthorized reproduction of this article is prohibited. 
where $G$ is a centered Gaussian density and $B$ a Gaussian random variable. Proving this result involves using the diffusion approximation theorem [14] to get asymptotic expressions for specific propagator moments from which we can deduce the expression for the limit $\widetilde{a}(Z, s)$. Therefore, when we capture the pulse at its random arrival time we will see a pulse whose shape does not depend on the realization of the random medium. This shape is the original pulse shape convolved with a Gaussian pulse shaping kernel. Thus, the effects of the random medium fluctuations can be described in terms of a random travel time correction and an anomalous diffusion effect. By the same approach we can also prove that

$$
b^{\varepsilon}(0, s) \longrightarrow 0 .
$$

This result shows that in the case with a constant homogenized medium the reflected wave will be negligible in the small $\varepsilon$ limit. While the mathematical derivation of this "medium pulse shaping" result was first obtained in [6] and [26], it was first derived in the geophysical literature in [32] via a heuristic derivation. The approximation has since come to be known as the O'Doherty-Anstey (ODA) theory after the authors of the pioneering paper, which lead to a string of papers both in the mathematical and geophysical literature, reflecting its relevance. However, so far the problem has only been analyzed in short range media. Here we generalize to the situation with long range media and show that then we get a qualitatively different result. We explain in section 3.2 that in this case we have a strong travel time perturbation, as in the classic theory; however, in the case with long range media the pulse shape itself is stable.

3. Medium model and main result. In this section we investigate the propagation in a long range medium. We first describe the model in detail, and then we establish the main result of the paper.

3.1. Long range model. We assume $\gamma \in(0,1)$ and that $\nu$ can be written as $\nu(z)=T(m(z))$ for every $z$ where the following hold:

- $T$ is a continuous function which is strictly bounded by 1 in absolute value, odd, and increasing. Note that our analysis remains valid in the case with $T$ not being bounded; the boundedness by 1 is introduced to make the model physically pertinent: we recall that $1+\varepsilon^{\kappa} \nu$ is the compressibility with $\kappa \geq 0$.

- $m$ is a Gaussian process, centered, stationary and has a correlation function $r_{m}$ which has the following asymptotic property as $z$ goes to $\infty$ :

$$
r_{m}(z)=\mathbb{E}[m(0) m(z)] \sim c_{m} z^{-\gamma} .
$$

Note that therefore the medium fluctuations $\nu$ themselves are not Gaussian in general; their distribution is controlled via the choice of $T$.

The property (3.1) is the so-called long range property. Its main consequence is that the covariance function $r_{m}$ of $m$ is not absolutely integrable:

$$
\int_{0}^{\infty}\left|r_{m}(z)\right| d z=\infty .
$$

Hence, this situation is in dramatic contrast with the classical mixing (or short range) case. Indeed, a mixing process has an integrable covariance function [22]. Another important consequence of (3.1) regards the choice of scales. We are given the correlation length of the medium $\left(1 / \varepsilon^{2}\right)$, the amplitude $\left(\varepsilon^{\kappa}\right)$, and the rate of decorrelation 
$\left(r_{m}(z) \sim c_{m} / z^{\gamma}\right)$ of the random perturbations. Then, because our goal is to capture the behavior of the transmitted wave pulse and its interaction with the medium, we have to choose an appropriate size of the window to capture the critical wavelength interaction scale by taking $\tau=\gamma+\kappa$. We next explain this scaling choice. The propagator equation is essentially driven by the process $\varepsilon^{\kappa-\tau} m\left(z / \varepsilon^{2}\right)$, and, we shall see that indeed its analysis involves the study of the convergence of the antiderivative $w^{\varepsilon}$ of this process:

$$
w^{\varepsilon}(z)=\int_{0}^{z} \frac{1}{\varepsilon^{\tau-\kappa}} m\left(\frac{z^{\prime}}{\varepsilon^{2}}\right) d z^{\prime} .
$$

However, it is known [35], as recalled in Lemma 1 just below, that the appropriate scale then is $\gamma=\tau-\kappa$. This gives convergence to a fractional Brownian motion. Let $H \in(0,1)$; then fractional (one-dimensional) Brownian motion (fBm) with Hurst parameter $H$ is the centered Gaussian process $\left(B_{H}(z)\right)_{z \in \mathbb{R}}$ with covariance function

$$
\mathbb{E}\left[B_{H}\left(z_{1}\right) B_{H}\left(z_{2}\right)\right]=\frac{1}{2}\left\{\left|z_{1}\right|^{2 H}+\left|z_{2}\right|^{2 H}-\left|z_{1}-z_{2}\right|^{2 H}\right\} .
$$

We refer the reader to Samorodnitsky and Taqqu's book [35] for a good reference. For the process $w^{\varepsilon}$ we have the convergence given next.

Lemma 1. Let $H=(2-\gamma) / 2$. As $\varepsilon$ goes to 0 , the finite-dimensional distributions of $w^{\varepsilon}$ converge to those of the fractional Brownian motion $c_{H}^{\prime} B_{H}$, where $c_{H}^{\prime 2}=c_{m} H^{-1}(2 H-1)^{-1}$.

Now we give two examples of processes $m$ :

- Fractional white noise with Hurst parameter $H=(2-\gamma) / 2 \in(1 / 2,1)$ that can be defined as

$$
m(z)=B_{H}(z+1)-B_{H}(z),
$$

where $B_{H}$ is the $\mathrm{fBm}$ with Hurst parameter $H$.

- The (stationary) fractional Ornstein-Uhlenbeck process with index $H=(2-$ $\gamma) / 2$ defined by

$$
m(z)=B_{H}(z)-e^{-z} \int_{-\infty}^{z} e^{z^{\prime}} B_{H}\left(z^{\prime}\right) d z^{\prime},
$$

where $B_{H}$ is the fBm with Hurst parameter $H$. As in the case with fractional white noise with index $H$, the fractional Ornstein-Uhlenbeck process is continuous, Gaussian, stationary, and centered and satisfies (3.1).

Notice that here we presented two examples for $m$ in terms of an $\mathrm{fBm}$, but all results in this paper are true and proved under the general assumptions on $m$ presented above, in particular Gaussianity and slow correlation decay. Notice also that we shall consider here

$$
H \in(1 / 2,1) \text { so that } \gamma \in(0,1) \text {. }
$$

We next introduce some notation. We denote by $X$ a Gaussian, centered, and reduced random variable: $X \sim \mathcal{N}(0,1)$. Letting $\sigma_{0}=\sqrt{\mathbb{E}\left[m(0)^{2}\right]}$, we will need the Hermite development of the function $T\left(\sigma_{0} \times \cdot\right)$. We denote by $H_{k}$ the $k$ th Hermite polynomial and by $J(k)$ the $k$ th Hermite coefficient of the function $T\left(\sigma_{0} \times \cdot\right)$, that is to say, $J(k)=\mathbb{E}\left[T\left(\sigma_{0} X\right) H_{k}(X)\right]$. Thanks to the assumptions on $T$, we have $J(0)=0$ 
and $J(1) \neq 0$, so that the Hermite coefficient of $T$ is 1 . Therefore, we can write (see the appendix for more details about Hermite polynomials)

$$
T\left(\sigma_{0} \times X\right)=\sum_{k=1}^{\infty} \frac{J(k)}{k !} H_{k}(X) .
$$

We conclude this subsection by establishing the long range behavior of $\nu$.

Lemma 2. For $z \rightarrow \infty$ we have

$$
r_{\nu}(z):=\mathbb{E}[\nu(0) \nu(z)] \sim \frac{c_{\nu}}{z^{\gamma}},
$$

where $c_{\nu}=c_{m} J(1)^{2} / \sigma_{0}^{2}=c_{m} \mathbb{E}\left[X T\left(\sigma_{0} X\right)\right]^{2} / \sigma_{0}^{2}$.

Proof. In view of (3.5) we can write (using (A.1))

$$
\nu(z)=\sum_{k=1}^{\infty} \frac{J(k)}{k !} H_{k}\left(\frac{m(z)}{\sigma_{0}}\right),
$$

so that (using (A.3))

$$
\begin{aligned}
\mathbb{E}[\nu(0) \nu(z)] & =\sum_{k=1}^{\infty} \frac{J(k)^{2}}{(k !)^{2}} \mathbb{E}\left[H_{k}\left(\frac{m(0)}{\sigma_{0}}\right) H_{k}\left(\frac{m(z)}{\sigma_{0}}\right)\right] \\
& =\sum_{k=1}^{\infty} \frac{J(k)^{2}}{k ! \sigma_{0}^{2 k}} r_{m}(z)^{k} .
\end{aligned}
$$

Therefore, we need to study the limit of

$$
z^{\gamma} \mathbb{E}[\nu(0) \nu(z)]=\sum_{k=1}^{\infty} \frac{J(k)^{2}}{k ! \sigma_{0}^{2 k}} z^{\gamma} r_{m}(z)^{k} .
$$

Observe that for $k=1$ we have $z^{\gamma} r_{m}(z) \sim c_{m}$ as $z \rightarrow \infty$, and for $k>1$ we have $z^{\gamma} r_{m}(z)^{k} \rightarrow 0$. Moreover, we have the uniform upper bound for $z$ sufficiently large:

$$
\frac{J(k)^{2}}{k ! \sigma_{0}^{2 k}} z^{\gamma}\left|r_{m}(z)\right|^{k} \leq \frac{J(k)^{2}}{k !} .
$$

Using the fact that (by (A.2))

$$
\sum_{k=1}^{\infty} \frac{J(k)^{2}}{k !}<\infty
$$

the result now follows from the uniform convergence theorem.

3.2. Main result. Now we establish the main result of this paper. We shall see that the asymptotic behavior of the transmission coefficient is quite different in the long range case than in the short range case. Recall that we let $\tau=\gamma+\kappa>0$.

THEOREM 1. Under the above assumptions, as $\varepsilon$ goes to $0,\left\{a^{\varepsilon}(Z, s)\right\}_{s}$ converges in distribution in the space of continuous functions endowed with the uniform topology to the random process $\{\widetilde{a}(Z, s)\}_{\text {s }}$ that can be written as

$$
\widetilde{a}(Z, s)=f\left(s-\frac{c_{H}}{2} B_{H}(Z)\right),
$$

Copyright (c) by SIAM. Unauthorized reproduction of this article is prohibited. 
where $B_{H}$ is a fractional Brownian motion with Hurst parameter $H=(2-\gamma) / 2$ and $c_{H}^{2}=c_{\nu} H^{-1}(2 H-1)^{-1}$ with $c_{\nu}$ as introduced in Lemma 2. Moreover, the process $\left\{b^{\varepsilon}(0, s)\right\}_{s}$ converges to 0 .

Therefore, we see that the pulse is stable and does not undergo a deterministic evolution in time as in the short range case. In the short range case the evolution of the pulse shape and the randomization of the pulse travel-distance takes place on the same time scale. In the long range case with persistent medium fluctuations and slow decorrelation the evolution of the pulse shape takes place on a relatively slow time scale, and in Theorem 1 we observe only the randomization of the travel distance while the pulse shape is stable. In the long range case with $H>1 / 2$ the medium fluctuations are persistent and "smoother" than in the classic case, so that the traveltime perturbation corresponding to an accumulation of fluctuations effect becomes relatively stronger than the pulse transformation effect which is due to scattering and enhanced by the roughness of the medium. Note that the traveltime perturbation is in the long range case described by a fractional Brownian motion with a Hurst index that corresponds to the effective Hurst index for the medium perturbations, while in the classic case it is described by a standard Brownian motion.

From the point of view of modern applications of the theory of waves in random media the above result is relevant. Recently there has been a lot of interest in imaging schemes in the context of cluttered layered media $[3,14]$ exploiting the ODA approximation, for instance, as well as in nonlayered media [4]. This reflects the fact that classic imaging schemes deteriorates when the "background" medium becomes fluctuating. The above results show how this body of results applies to the long range situation, in which case the modeling of the travel time perturbation becomes the important aspect. We remark also that currently there is a lot of interest in the design of robust wireless communication schemes when the signalling takes place through clutter, through the turbulent atmosphere, for instance, which is relevant also in the context of remote sensing. Design of robust schemes requires a forward model that captures the interaction of the pulse with the medium, which in the long range persistent and layered case is described by Theorem 1 . The derivation of this result, which is presented in section 5 , sets forth a framework which we expect will be useful also in a more general context to describe other physical scaling scenarios, analogous to the case with short range medium fluctuations $[17,18]$.

4. Numerical illustration. We illustrate the results with some numerical simulations. In the numerical simulations we use a Gaussian initial pulse shape. In the normalized coordinates the support of the initial pulse is $10^{-3}$ and the total propagation distance is 1 . The medium is defined as in (3.2) with $\kappa=2 H$ and, moreover, with $\epsilon=10^{-2}$ and with a cutoff function that is the identity in the neighborhood of the origin with a smooth cutoff. We use a discretization corresponding to equal travel time sections and the method described in [33] to simulate the realizations of the medium fluctuations. In Figure 4.1 we show the result of three simulations when the propagated pulse is plotted relative to its random arrival time when $H=.6$ and on the fine scale $\varepsilon^{\tau}$. Observe that indeed the pulse shape is to leading order not affected by random fluctuations in the medium, as predicted by Theorem 1. In Figure 4.2 we show the corresponding picture when $H=1 / 2$. Note that in this case the pulse shape is modified via a convolution with a Gaussian kernel as described by the classical pulse shaping or ODA theory in the case of strong mixing.

5. Proof of Theorem 1. We first give an outline of the proof. As recalled in section 2 the process $\left\{a^{\varepsilon}(Z, s)\right\}_{s}$ can be written in terms of the propagator $P_{\omega}^{\varepsilon}$, and 


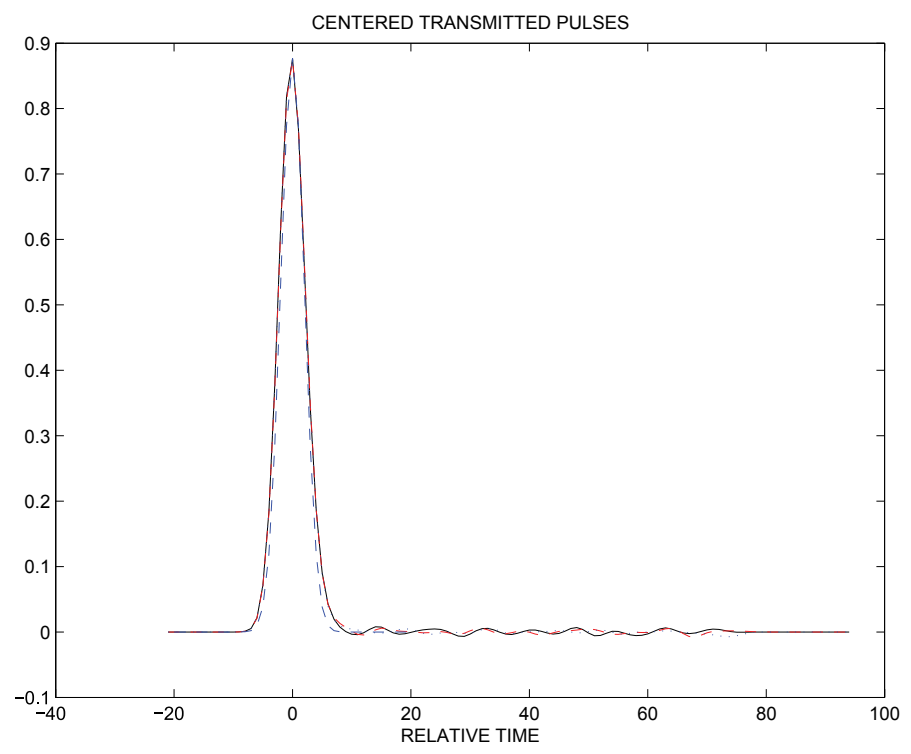

FIG. 4.1. The transmitted wave shown at a fixed depth for several medium realizations and with $H=.6$. The dashed line is the original pulse shape.

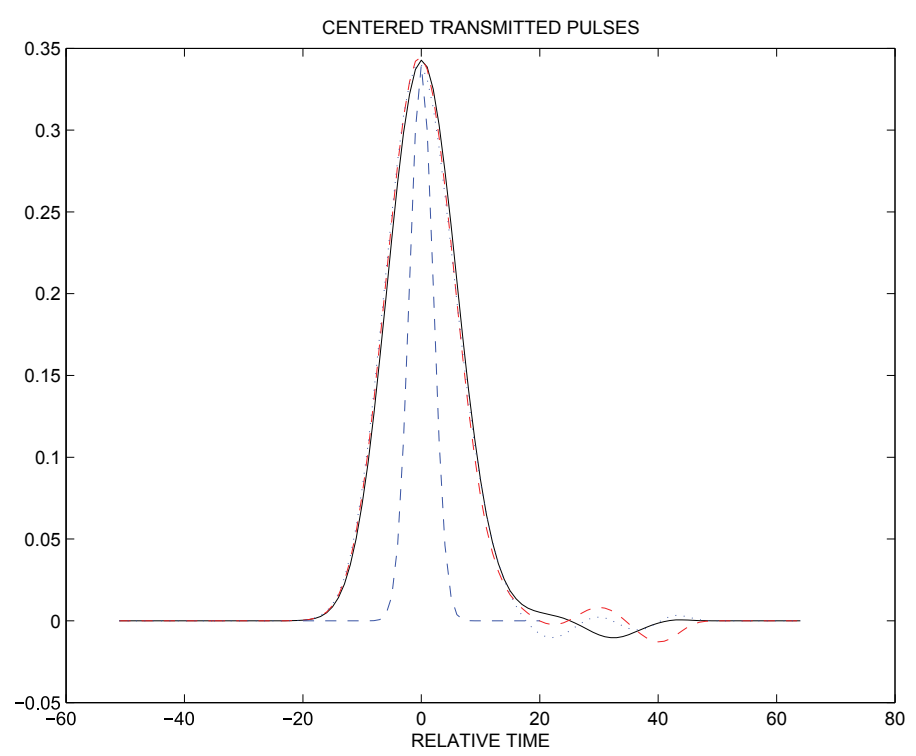

FIG. 4.2. The transmitted wave shown at a fixed depth for several medium realizations and with $H=1 / 2$. The dashed line is the original pulse shape.

thus the study of the convergence of $\left\{a^{\varepsilon}(Z, s)\right\}_{s}$ can be analyzed via asymptotic properties of $P_{\omega}^{\varepsilon}$. This convergence analysis will follow the lines of [30,31]. The propagator $P_{\omega}^{\varepsilon}$ satisfies the equation

$$
\frac{d P_{\omega}^{\varepsilon}}{d z}(z)=\frac{1}{\varepsilon^{\gamma}} H_{\omega}\left(\frac{z}{\varepsilon^{\tau}}, \frac{z}{\varepsilon^{2}}\right) P_{\omega}^{\varepsilon}(z)
$$

Copyright $@$ by SIAM. Unauthorized reproduction of this article is prohibited. 
which we can write in the form

$$
d P_{\omega}^{\varepsilon}(z)=\frac{i \omega}{2} \sum_{j=1}^{3} F_{j} P_{\omega}^{\varepsilon} d v_{j}^{\varepsilon}(z)
$$

where

$$
F_{1}=\left(\begin{array}{cc}
1 & 0 \\
0 & -1
\end{array}\right), \quad F_{2}=\left(\begin{array}{cc}
0 & -1 \\
1 & 0
\end{array}\right), \quad \text { and } \quad F_{3}=\left(\begin{array}{cc}
0 & i \\
i & 0
\end{array}\right),
$$

and $v_{1}^{\varepsilon}, v_{2}^{\varepsilon}$, and $v_{3}^{\varepsilon}$ are three processes of bounded variation that we can write as

$$
\begin{aligned}
& v_{1}^{\varepsilon}(z)=\int_{0}^{z} \frac{1}{\varepsilon^{\gamma}} \nu\left(\frac{z^{\prime}}{\varepsilon^{2}}\right) d z^{\prime}, \\
& v_{2}^{\varepsilon}(z)=\int_{0}^{z} \frac{1}{\varepsilon^{\gamma}} \nu\left(\frac{z^{\prime}}{\varepsilon^{2}}\right) \cos \left(2 \omega \frac{z^{\prime}}{\varepsilon^{\tau}}\right) d z^{\prime}, \\
& v_{3}^{\varepsilon}(z)=\int_{0}^{z} \frac{1}{\varepsilon^{\gamma}} \nu\left(\frac{z^{\prime}}{\varepsilon^{2}}\right) \sin \left(2 \omega \frac{z^{\prime}}{\varepsilon^{\tau}}\right) d z^{\prime} .
\end{aligned}
$$

Thanks to Lyons's rough paths theory for which we recall some tools in section 5.1, we shall see that the convergence of $P_{\omega}^{\varepsilon}$ can be reduced for a convenient topology to the convergence of the process $\mathbf{v}^{\varepsilon}$ defined as

$$
\mathbf{v}^{\varepsilon}:=\left(v_{1}^{\varepsilon}, v_{2}^{\varepsilon}, v_{3}^{\varepsilon}\right) .
$$

Hence, we first prove the convergence of $\mathbf{v}^{\varepsilon}$, then by Theorem 2 below we deduce the convergence of $P_{\omega}^{\varepsilon}$, and thanks to (2.12) we finally conclude by the convergence of $\left\{a^{\varepsilon}(Z, s)\right\}_{s}$.

5.1. Rough paths. In this section we fix $p \in[1,2)$ and consider a closed interval $I=[0, Z]$. We define the $p$-variation of a continuous function $w: I \rightarrow \mathbb{R}^{n}$ by

$$
V_{p}(w):=\left(\sup _{D} \sum_{j=0}^{k-1}\left\|w\left(z_{j+1}\right)-w\left(z_{j}\right)\right\|^{p}\right)^{1 / p},
$$

where $\sup _{D}$ runs over all finite partitions $\left\{0=z_{0}, \ldots, z_{k}=Z\right\}$ of $I$ and where here and below $\|\cdot\|$ refers to the Euclidean norm. The space of all continuous functions of bounded variation (1-variation) is endowed with the $p$-variation distance

$$
\|w\|_{p}=V_{p}(w)+\sup _{z \in[0,1]}|w(z)|
$$

and is denoted by $\Omega_{p}^{\infty}$. The closure of this metric space is called the space of all geometric rough paths and is denoted by $\Omega_{p}$. One of the most important theorems of rough paths theory is the following.

TheOREM 2 (Lyons's continuity theorem [27]). Let ${ }^{1} G: \mathbb{R} \times \mathbb{R}^{d} \rightarrow \mathcal{L}\left(\mathbb{R}, \mathbb{R}^{d}\right)$ and $F: \mathbb{R} \times \mathbb{R}^{d} \rightarrow \mathcal{L}\left(\mathbb{R}^{n}, \mathbb{R}^{d}\right)$ be two smooth functions. Let $y$ be the unique solution of the differential equation

$$
d y(z)=G(z, y(z)) d z+F(z, y(z)) d w(z), \quad y(z=0)=y_{0},
$$

\footnotetext{
${ }^{1}$ Here $\mathcal{L}\left(\mathbb{R}, \mathbb{R}^{d}\right)$ (resp., $\mathcal{L}\left(\mathbb{R}^{n}, \mathbb{R}^{d}\right)$ ) denotes the space of all linear maps from $\mathbb{R}$ (resp., $\mathbb{R}^{n}$ ) to $\mathbb{R}^{d}$
} 
where $w$ is a bounded variation function. Then the Itô map $\mathcal{I}: w \mapsto y$ is continuous with respect to the p-variation distance from $\Omega_{p}^{\infty}\left(\mathbb{R}^{n}\right)$ to $\Omega_{p}^{\infty}\left(\mathbb{R}^{d}\right)$. Therefore there exists a unique extension of this map (that we still denote by $\mathcal{I}$ ) to the space $\Omega_{p}\left(\mathbb{R}^{n}\right)$.

This theorem has been proved by Lyons and extensively studied and applied (see $[8,23,24,27,28,29])$.

The proof of Theorem 1 is based on analysis of the tightness in the space of geometric rough paths. In the context of this we need to compute the $p$-variation for $p>1$. To this effect we will need the following lemmas, of which the first can be found, for instance, in [24] and the second in [23, 24].

LEMMA 3. Let $q \in[1,2)$ and $\left(v^{\varepsilon}\right)_{\varepsilon>0}$ be a family of continuous random processes of finite q-variation which is tight in the space of continuous functions on $I$ and satisfies

$$
\lim _{A \rightarrow+\infty} \sup _{\varepsilon>0} \mathbb{P}\left[V_{q}\left(v^{\varepsilon}\right)>A\right]=0 .
$$

Then $\left(v^{\varepsilon}\right)_{\varepsilon>0}$ is tight in $\Omega_{p}$ for every $p>q$.

Lemma 4. For every $n \in \mathbb{N}$ and every $k=0,1, \ldots, 2^{n}$, we let $z_{k}^{n}:=Z k / 2^{n}$. Let $q \in[1,2)$ and $v$ be a function of finite q-variation. Then there exist two positive constants $C_{1}, C_{2}$ which do not depend on $v$ such that

$$
V_{q}(v)^{q} \leq C_{1} \sum_{n=1}^{+\infty} n^{C_{2}} \sum_{k=1}^{2^{n}}\left\|v\left(z_{k}^{n}\right)-v\left(z_{k-1}^{n}\right)\right\|^{q} .
$$

We conclude this subsection by mentioning an application of this theory to fractional Brownian motion introduced in section 3.1. From the definition of $\mathrm{fBm} B_{H}$ with index $H$ we remark that if $H=1 / 2$, the process $B_{H}$ is the classical Brownian motion $(\mathrm{cBm})$. However, if $H \neq 1 / 2, W_{H}$ is neither a semimartingale nor a Markov process. As a consequence, the construction for the $\mathrm{fBm}$ of a stochastic calculus turns out to be more involved than for the $\mathrm{cBm}$. This can be done by several way [7], and here we use the rough paths approach as in [8].

5.2. Convergence of the propagator. Using Theorem 2 and the expression (5.1), the asymptotic study of the propagator is reduced to finding the limit in a rough path space of $\mathbf{v}^{\varepsilon}:=\left(v_{1}^{\varepsilon}, v_{2}^{\varepsilon}, v_{3}^{\varepsilon}\right)$. This is the subject of the following lemma.

LEMMA 5. Let $p>2 /(2-\gamma) \equiv 1 / H$. As $\varepsilon$ goes to 0 , the increments of $\mathbf{v}^{\varepsilon}$ converge in $\Omega_{p}$ to those of $W_{H}$, which can be written as

$$
W_{H}=\left(c_{H} B_{H}, 0,0\right),
$$

where $B_{H}$ is a fractional Brownian motion with Hurst parameter $H=(2-\gamma) / 2$.

The proof of Lemma 5 is based on establishing several technical lemmas that we derive next. Below we will repeatedly use the notation

$$
w^{\varepsilon}(z)=\int_{0}^{z} \frac{1}{\varepsilon^{\gamma}} m\left(\frac{z^{\prime}}{\varepsilon^{2}}\right) d z^{\prime} .
$$

We consider first $v_{1}^{\varepsilon}$.

Lemma 6. As $\varepsilon$ goes to 0 , the finite-dimensional distributions of $v_{1}^{\varepsilon}$ converge to those of the fractional Brownian motion $c_{H} B_{H}$ with $c_{H}^{2}=c_{\nu} H^{-1}(2 H-1)^{-1}$.

Lemma 6 is a continuous version of [40]. Moreover, a stronger version of this result was established in [41]. Nevertheless, we present here a (simple) proof of Lemma 6 for the sake of completeness.

Copyright $@$ by SIAM. Unauthorized reproduction of this article is prohibited. 
Proof. In view of Lemma 1 it is enough to show that

$$
\lim _{\varepsilon \rightarrow 0} \mathbb{E}\left[\left|v_{1}^{\varepsilon}(z)-\sqrt{\frac{c_{\nu}}{c_{m}}} w^{\varepsilon}(z)\right|^{2}\right]=0 .
$$

Consider the development of $T\left(\sigma_{0} \times \cdot\right)$ in the base of Hermite polynomials $\left\{H_{j}\right\}_{j=0,1, \ldots \text {, }}$

$$
T(m)=\sum_{j=1}^{\infty} \frac{J(j)}{j !} H_{j}\left(\frac{m}{\sigma_{0}}\right),
$$

and observe that $J(1) / \sigma_{0}=\sqrt{c_{\nu} / c_{m}}$; by (A.3) we then have

$$
\begin{aligned}
& \mathbb{E}\left[\left|v_{1}^{\varepsilon}(z)-\sqrt{\frac{c_{\nu}}{c_{m}}} w^{\varepsilon}(z)\right|^{2}\right]=\mathbb{E}\left[\left|\varepsilon^{2 H} \int_{0}^{z / \varepsilon^{2}} \sum_{j=2}^{\infty} \frac{J(j)}{j !} H_{j}\left(\frac{m(x)}{\sigma_{0}}\right) d x\right|^{2}\right] \\
& =\varepsilon^{4 H} \int_{0}^{z / \varepsilon^{2}} d x \int_{0}^{z / \varepsilon^{2}} d y \sum_{j=2}^{\infty}\left(\frac{J(j)}{j !}\right)^{2} \mathbb{E}\left[H_{j}\left(\frac{m(x)}{\sigma_{0}}\right) H_{j}\left(\frac{m(y)}{\sigma_{0}}\right)\right] \\
& =\varepsilon^{4 H} \int_{0}^{z / \varepsilon^{2}} d x \int_{0}^{z / \varepsilon^{2}} d y \sum_{j=2}^{\infty} \frac{J(j)^{2}}{j !}\left(\frac{r(x-y)}{\sigma_{0}^{2}}\right)^{j} \\
& \leq \varepsilon^{4 H} \int_{0}^{z / \varepsilon^{2}} d x \int_{0}^{z / \varepsilon^{2}} d y \sum_{j=2}^{\infty} \frac{J(j)^{2}}{j !}\left(\frac{r(x-y)}{\sigma_{0}^{2}}\right)^{2} \\
& \leq \varepsilon^{4 H} C^{\prime} \int_{0}^{z / \varepsilon^{2}} d x \int_{0}^{z / \varepsilon^{2}} d y r(x-y)^{2},
\end{aligned}
$$

with

$$
C^{\prime}=\sum_{j=2}^{\infty} \frac{J(j)^{2}}{j ! \sigma_{0}^{4}}<\infty
$$

As $u \rightarrow \infty$, we have $r(u) \sim c u^{-\gamma}$; therefore for every $\eta>0$ there exist $z_{\eta}, C_{\eta}$, and $\tilde{C}_{\eta}$ such that

$$
\begin{aligned}
\varepsilon^{4 H} \int_{0}^{z / \varepsilon^{2}} d x \int_{0}^{z / \varepsilon^{2}} d y r(x-y)^{2} \leq & \varepsilon^{4 H} \sigma_{0}^{4} \int_{0}^{z / \varepsilon^{2}} d x \int_{0}^{z / \varepsilon^{2}} d y 1_{|x-y| \leq z_{\eta}} \\
& +\varepsilon^{4 H} \eta \int_{0}^{z / \varepsilon^{2}} d x \int_{0}^{z / \varepsilon^{2}} d y|r(x-y)| \\
\leq & \varepsilon^{4 H-2} C_{\eta}+\eta|z|^{2-\gamma} \tilde{C}_{\eta}
\end{aligned}
$$

Then

$$
\limsup _{\varepsilon \rightarrow 0} \mathbb{E}\left[\left|v_{1}^{\varepsilon}(z)-\sqrt{\frac{c_{\nu}}{c_{m}}} w^{\varepsilon}(z)\right|^{2}\right] \leq \eta|z|^{2-\gamma} \tilde{C}_{\eta}
$$

for every $\eta>0$, which concludes the proof.

We consider next $v_{2}^{\varepsilon}$ and $v_{3}^{\varepsilon}$.

Lemma 7. For every $z \in[0, Z]$, as $\varepsilon$ goes to 0 , the finite-dimensional distributions of $v_{2}^{\varepsilon}(z)$ and $v_{3}^{\varepsilon}(z)$ converge to those of the 0 process.

Copyright $@$ by SIAM. Unauthorized reproduction of this article is prohibited. 
Proof. Without loss of generality we present the proof only for $v_{2}^{\varepsilon}(z)$ and with $2 \omega=1$. We have

$$
\begin{aligned}
\mathbb{E}\left[v_{2}^{\varepsilon}(z)^{2}\right] & =\frac{1}{\varepsilon^{2 \gamma}} \int_{0}^{z} d x \int_{0}^{z} d y \cos \left(\frac{x}{\varepsilon^{\tau}}\right) \cos \left(\frac{y}{\varepsilon^{\tau}}\right) r_{\nu}\left(\frac{x-y}{\varepsilon^{2}}\right) \\
& =I_{1}^{\varepsilon}(z)+I_{2}^{\varepsilon}(z),
\end{aligned}
$$

with

$$
\begin{aligned}
& I_{1}^{\varepsilon}(z)=\frac{c_{\nu}}{\varepsilon^{2 \gamma}} \int_{0}^{z} d x \int_{0}^{z} d y \cos \left(\frac{x}{\varepsilon^{\tau}}\right) \cos \left(\frac{y}{\varepsilon^{\tau}}\right)\left|\frac{x-y}{\varepsilon^{2}}\right|^{-\gamma} \\
& I_{2}^{\varepsilon}(z)=\frac{1}{\varepsilon^{2 \gamma}} \int_{0}^{z} d x \int_{0}^{z} d y \cos \left(\frac{x}{\varepsilon^{\tau}}\right) \cos \left(\frac{y}{\varepsilon^{\tau}}\right)\left(r_{\nu}\left(\frac{x-y}{\varepsilon^{2}}\right)-c_{\nu}\left|\frac{x-y}{\varepsilon^{2}}\right|^{-\gamma}\right) .
\end{aligned}
$$

Let $\delta>0$; because $r_{\nu}(u) \sim c_{\nu} u^{-\gamma}$ as $u \rightarrow \infty$, we have that for $u>z_{\delta}$ (with $z_{\delta}$ sufficiently large) $\left|r_{\nu}(u)-c_{\nu} u^{-\gamma}\right| \leq \delta u^{-\gamma}$. We then obtain

$$
\begin{aligned}
\left|I_{2}^{\varepsilon}(z)\right| \leq & \frac{\delta}{\varepsilon^{2 \gamma}} \int_{0}^{z} d x \int_{0}^{z} d y \cos \left(\frac{x}{\varepsilon^{\tau}}\right) \cos \left(\frac{y}{\varepsilon^{\tau}}\right)\left|\frac{x-y}{\varepsilon^{2}}\right|^{-\gamma} \\
& +C \int_{0}^{z} d x \int_{0}^{z} d y|x-y|^{-\gamma} 1_{|x-y| \leq \varepsilon^{2} z_{\delta}} \\
\leq & \delta \int_{0}^{z} d x \int_{0}^{z} d y|x-y|^{-\gamma}+C \int_{0}^{z} d x \int_{0}^{z} d y|x-y|^{-\gamma} 1_{|x-y| \leq \varepsilon^{2} z_{\delta}},
\end{aligned}
$$

so that

$$
\limsup _{\varepsilon \rightarrow 0}\left|I_{2}^{\varepsilon}(z)\right| \leq \delta \int_{0}^{z} d x \int_{0}^{z} d y|x-y|^{-\gamma} .
$$

The inequality above is valid for every $\delta>0$, and we conclude

$$
\lim _{\varepsilon \rightarrow 0} I_{2}^{\varepsilon}(z)=0 .
$$

To complete the study of $v_{2}^{\varepsilon}$ it remains to deal with $I_{1}^{\varepsilon}(z)$. We have

$$
\begin{aligned}
I_{1}^{\varepsilon}(z) & =c_{\nu} \varepsilon^{4-2 \gamma} \int_{0}^{z / \varepsilon^{2}} d x \int_{0}^{z / \varepsilon^{2}} d y \cos \left(\varepsilon^{2-\tau} x\right) \cos \left(\varepsilon^{2-\tau} y\right)|x-y|^{-\gamma} \\
& =\frac{c_{\nu}}{2}\left(I_{1,1}^{\varepsilon}(z)+I_{1,2}^{\varepsilon}(z)\right)
\end{aligned}
$$

where

$$
\begin{aligned}
& I_{1,1}^{\varepsilon}(z)=\varepsilon^{4-2 \gamma} \int_{0}^{z / \varepsilon^{2}} d x \int_{0}^{z / \varepsilon^{2}} d y \cos \left(\varepsilon^{2-\tau}(x-y)\right)|x-y|^{-\gamma}, \\
& I_{1,2}^{\varepsilon}(z)=\varepsilon^{4-2 \gamma} \int_{0}^{z / \varepsilon^{2}} d x \int_{0}^{z / \varepsilon^{2}} d y \cos \left(\varepsilon^{2-\tau}(x+y)\right)|x-y|^{-\gamma} .
\end{aligned}
$$

Using integration by parts, we get

$$
I_{1,1}^{\varepsilon}(z)=2\left(\varepsilon^{4-2 \gamma} \int_{0}^{z / \varepsilon^{2}} d x\left(\int_{0}^{(z-x) / \varepsilon^{2}} d y \cos \left(\varepsilon^{2-\tau} y\right)|y|^{-\gamma}\right)\right)=2\left(J_{1}^{\varepsilon}(z)-J_{2}^{\varepsilon}(z)\right),
$$


where

$$
\begin{aligned}
& J_{1}^{\varepsilon}(z)=\varepsilon^{2-2 \gamma} z \int_{0}^{z / \varepsilon^{2}} d y \cos \left(\varepsilon^{2-\tau} y\right) y^{-\gamma}, \\
& J_{2}^{\varepsilon}(z)=\varepsilon^{4-2 \gamma} \int_{0}^{z / \varepsilon^{2}} d y \cos \left(\varepsilon^{2-\tau} y\right) y^{1-\gamma} .
\end{aligned}
$$

We make the substitution $y \rightarrow y / \varepsilon^{2-\tau}$ in $J_{1}^{\varepsilon}(z)$ to obtain

$$
J_{1}^{\varepsilon}(z)=\varepsilon^{\tau(1-\gamma)} z \int_{0}^{z / \varepsilon^{\tau}} d y \cos (y) y^{-\gamma}
$$

and so $J_{1}^{\varepsilon}(z)=O\left(\varepsilon^{\tau(1-\gamma)}\right)$. We also make the substitution $y \rightarrow y / \varepsilon^{2-\tau}$ in $J_{2}^{\varepsilon}(z)$ to obtain

$$
J_{2}^{\varepsilon}(z)=\varepsilon^{\tau(2-\gamma)} \int_{0}^{z / \varepsilon^{\tau}} d y \cos (y) y^{1-\gamma} .
$$

Using integration by parts, we obtain

$$
\begin{aligned}
J_{2}^{\varepsilon}(z)= & \varepsilon^{\tau(2-\gamma)} \sin \left(\frac{z}{\varepsilon^{\tau}}\right)\left(\frac{z}{\varepsilon^{\tau}}\right)^{1-\gamma} \\
& -\varepsilon^{\tau(2-\gamma)}(1-\gamma) \int_{0}^{z / \varepsilon^{\tau}} d y \sin (y) y^{-\gamma},
\end{aligned}
$$

and so $J_{2}^{\varepsilon}(z)=O\left(\varepsilon^{\tau}\right)$. Therefore, we conclude

$$
\lim _{\varepsilon \rightarrow 0} I_{1,1}^{\varepsilon}(z)=0 .
$$

Consider finally $I_{1,2}^{\varepsilon}(z)$. Letting $x-y \rightarrow x$ and $x+y \rightarrow y$, we get

$$
I_{1,2}^{\varepsilon}(s, t)=\varepsilon^{4-2 \gamma} \iint_{D_{1}^{\varepsilon} \cup D_{2}^{\varepsilon} \cup D_{3}^{\varepsilon} \cup D_{4}^{\varepsilon}} d x d y|x|^{-\gamma} \frac{\cos \left(\varepsilon^{2-\tau} y\right)}{2},
$$

where

$$
\begin{aligned}
& D_{1}^{\varepsilon}=\left\{(x, y) \in\left[0, z / \varepsilon^{2}\right] \times\left[0, z / \varepsilon^{2}\right]: x \leq y\right\}, \\
& D_{2}^{\varepsilon}=\left\{(x, y) \in\left[0, z / \varepsilon^{2}\right] \times\left[z / \varepsilon^{2}, 2 z / \varepsilon^{2}\right]: y \leq-x+2 z / \varepsilon^{2}\right\}, \\
& D_{3}^{\varepsilon}=\left\{(x, y) \in\left[-z / \varepsilon^{2}, 0\right] \times\left[z / \varepsilon^{2}, 2 z / \varepsilon^{2}\right]: y \leq x+2 z / \varepsilon^{2}\right\}, \\
& D_{4}^{\varepsilon}=\left\{(x, y) \in\left[-z / \varepsilon^{2}, 0\right] \times\left[0, z / \varepsilon^{2}\right]:-y \leq x\right\} .
\end{aligned}
$$

Let us deal with the integral on $D_{1}^{\varepsilon}$ :

$$
\varepsilon^{4-2 \gamma} \iint_{D_{1}^{\varepsilon}} d x d y|x|^{-\gamma} \frac{\cos \left(\varepsilon^{2-\tau} y\right)}{2}=(2(1-\gamma))^{-1} J_{2}^{\varepsilon}(z)=O\left(\varepsilon^{\tau}\right) .
$$

The integrals on $D_{2}^{\varepsilon}, D_{3}^{\varepsilon}$, and $D_{4}^{\varepsilon}$ can be analyzed in a similar way; therefore, $I_{1,2}^{\varepsilon}(s, t) \rightarrow$ 0 . This finally shows

$$
\lim _{\varepsilon \rightarrow 0} I_{1}^{\varepsilon}(z)=0,
$$

Copyright (c) by SIAM. Unauthorized reproduction of this article is prohibited. 
and then

$$
\lim _{\varepsilon \rightarrow 0} \mathbb{E}\left[v_{2}^{\varepsilon}(z)^{2}\right]=0
$$

which concludes the proof.

Now we deal with a technical lemma regarding increments of $\mathbf{v}^{\varepsilon}$.

LEMma 8. There exists a constant $C>0$ such that for every $z, \zeta$, and $\varepsilon>0$ we have

$$
\mathbb{E}\left[\left\|\mathbf{v}^{\varepsilon}(z)-\mathbf{v}^{\varepsilon}(\zeta)\right\|^{2}\right] \leq C|z-\zeta|^{2 H}
$$

Proof. For every $j=1,2,3$, using (3.6), (3.7), and (A.3), we have (taking $z>\zeta$ )

$$
\begin{aligned}
& \mathbb{E}\left[\left|v_{j}^{\varepsilon}(z)-v_{j}^{\varepsilon}(\zeta)\right|^{2}\right] \leq \frac{1}{\varepsilon^{2 \gamma}} \int_{\zeta}^{z} d x \int_{\zeta}^{z} d y\left|\mathbb{E}\left[\nu\left(\frac{x}{\varepsilon^{2}}\right) \nu\left(\frac{y}{\varepsilon^{2}}\right)\right]\right| \\
& \leq \frac{C}{\varepsilon^{2 \gamma}} \int_{\zeta}^{z} d x \int_{\zeta}^{z} d y \sum_{j=1}^{\infty}\left(\frac{J(j)}{j !}\right)^{2}\left|\mathbb{E}\left[H_{j}\left(\frac{m}{\sigma_{0}}\left(\frac{x}{\varepsilon^{2}}\right)\right) H_{j}\left(\frac{m}{\sigma_{0}}\left(\frac{y}{\varepsilon^{2}}\right)\right)\right]\right| \\
& \quad \leq \frac{C}{\varepsilon^{2 \gamma}} \int_{\zeta}^{z} d x \int_{\zeta}^{z} d y \sum_{j=1}^{\infty} \frac{J(j)^{2}}{j ! \sigma_{0}^{2 j}}\left|r_{m}\left(\frac{x-y}{\varepsilon^{2}}\right)\right|^{j} \\
& \quad \leq \frac{C}{\varepsilon^{2 \gamma}} \int_{\zeta}^{z} d x \int_{\zeta}^{z} d y \sum_{j=1}^{\infty} \frac{J(j)^{2}}{j ! \sigma_{0}^{2}}\left|r_{m}\left(\frac{x-y}{\varepsilon^{2}}\right)\right| \\
& \leq \frac{C^{\prime}}{\varepsilon^{2 \gamma}} \int_{\zeta}^{z} d x \int_{\zeta}^{z} d y\left|\frac{x-y}{\varepsilon^{2}}\right|^{-\gamma} \\
& \leq \frac{2 C^{\prime}}{(1-\gamma)(2-\gamma)}|z-\zeta|^{2-\gamma},
\end{aligned}
$$

which concludes the proof.

Using the above lemmas, we can deduce the following lemma, which deals with identification of the limit.

Lemma 9. As $\varepsilon$ goes to $0, \mathbf{v}^{\varepsilon}$ converges to $W_{H}$ defined in Lemma 5 in the space of continuous functions endowed with the uniform norm.

Proof. Lemmas 6 and 7 give the convergence of finite-dimensional distributions of $\mathbf{v}^{\varepsilon}$ to those of $W_{H}$. Using then the Kolmogorov criterion [2], Lemma 8, and the fact that $2 H>1$, we get the tightness of $\left(\mathbf{v}^{\varepsilon}\right)_{\varepsilon}$ in the space of continuous functions endowed with the uniform norm, which establishes the proof.

Thanks to Lemma 9 we conclude the proof of Lemma 5 by establishing the tightness in a rough paths sense.

Lemma 10. The sequence $\left(\mathbf{v}^{\varepsilon}\right)_{\varepsilon}$ is tight in $\Omega_{p}$ for $p>1 / H$.

Proof of Lemmas 5 and 10 . Let $q \in(1 / H, p)$. In view of Lemmas 3 and 9 it is enough to prove

$$
\lim _{A \rightarrow+\infty} \sup _{\varepsilon>0} \mathbb{P}\left[V_{q}\left(\mathbf{v}^{\varepsilon}\right)>A\right]=0
$$

Copyright (C) by SIAM. Unauthorized reproduction of this article is prohibited. 
Using Chebyshev's inequality, the fact that $q<2$, Lemma 4, the Hölder inequality, and Lemma 9, we find

$$
\begin{aligned}
\mathbb{P}\left[V_{q}\left(\mathbf{v}^{\varepsilon}\right)>A\right] & \leq \frac{1}{A^{q}} \mathbb{E}\left[V_{q}\left(\mathbf{v}^{\varepsilon}\right)^{q}\right] \\
& \leq \frac{C}{A^{q}} \sum_{n=1}^{+\infty} n^{C} \sum_{k=1}^{2^{n}} \mathbb{E}\left[\left\|\mathbf{v}^{\varepsilon}\left(z_{k}^{n}\right)-\mathbf{v}^{\varepsilon}\left(z_{k-1}^{n}\right)\right\|^{q}\right] \\
& \leq \frac{C}{A^{q}} \sum_{n=1}^{+\infty} n^{C} \sum_{k=1}^{2^{n}} \mathbb{E}\left[\left\|\mathbf{v}^{\varepsilon}\left(z_{k}^{n}\right)-\mathbf{v}^{\varepsilon}\left(z_{k-1}^{n}\right)\right\|^{2}\right]^{q / 2} \\
& \leq \frac{C^{\prime}}{A^{q}} \sum_{n=1}^{+\infty} n^{C} \sum_{k=1}^{2^{n}}\left(\frac{1}{2^{n}}\right)^{q H} \\
& \leq \frac{C^{\prime}}{A^{q}} \sum_{n=1}^{+\infty} n^{C}\left(\frac{1}{2^{n}}\right)^{q H-1}
\end{aligned}
$$

and since $q H>1$ we deduce (5.4).

Finally, we can now derive the following lemma, which deals with the convergence of the propagator.

Lemma 11. Let $\left\{\omega_{1}, \ldots, \omega_{n}\right\}$ be a collection of frequencies. Then, as $\varepsilon$ goes to 0 , the propagator vector $\left(P_{\omega_{1}}^{\varepsilon}, \ldots, P_{\omega_{n}}^{\varepsilon}\right)$ converges to $\left(P_{\omega_{1}}, \ldots, P_{\omega_{n}}\right)$, which is the asymptotic propagator $P_{\omega}$ that we can write as

$$
P_{\omega}(z)=\left(\begin{array}{cc}
\exp \left(i \omega c_{H} / 2 B_{H}(z)\right) & 0 \\
0 & \exp \left(-i \omega c_{H} / 2 B_{H}(z)\right)
\end{array}\right)
$$

Proof. By combining Theorem 2, (5.1), and Lemma 5, we get that, as $\varepsilon$ goes to 0 , $P_{\omega}^{\varepsilon}$ converges in distribution in the space of continuous functions (endowed with the uniform topology) to the solution $P_{\omega}$ of the following system of equations in the sense of rough paths:

$$
d P_{\omega}(z)=\frac{i \omega c_{H}}{2}\left(\begin{array}{cc}
1 & 0 \\
0 & -1
\end{array}\right) P_{\omega}(z) d B_{H}(z) .
$$

This concludes the proof.

5.3. Conclusion of the proof. The remaining part of the proof of Theorem 1 follows the lines of $[6,14]$; however, we present it here for completeness. Recall that, thanks to the formula (2.12), we can write $a^{\varepsilon}(Z, s)$ in a Fourier-type formula using the transmission coefficient,

$$
a^{\varepsilon}(Z, s)=\frac{1}{2 \pi} \int e^{-i s \omega} T_{\omega}^{\varepsilon}(Z) \widehat{f}(\omega) d \omega,
$$

with the transmission coefficient being a functional of the propagator $P_{\omega}^{\varepsilon}$. We shall use Lemma 11 to deduce the convergence of the transmitted wave.

Let $n \in \mathbb{N}, s_{1} \leq \cdots \leq s_{n} \in[0, \infty)$. We can write

$$
\begin{aligned}
& \mathbb{E}\left[a^{\varepsilon}\left(Z, s_{1}\right) \cdots a^{\varepsilon}\left(Z, s_{n}\right)\right]=\mathbb{E}\left[\frac{1}{(2 \pi)^{n}} \prod_{j=1}^{n} \int e^{-i s_{j} \omega} T_{\omega}^{\varepsilon}(Z) \widehat{f}(\omega) d \omega\right] \\
& =\frac{1}{(2 \pi)^{n}} \int \cdots \int e^{-i \sum_{j=1}^{n} s_{j} \omega_{j}} \widehat{f}\left(\omega_{1}\right) \cdots \widehat{f}\left(\omega_{n}\right) \mathbb{E}\left[T_{\omega_{1}}^{\varepsilon}(Z) \cdots T_{\omega_{n}}^{\varepsilon}(Z)\right] d \omega_{1} \cdots d \omega_{n} .
\end{aligned}
$$

Copyright (c) by SIAM. Unauthorized reproduction of this article is prohibited. 
Thanks to Lemma 11 we have that as $\varepsilon \rightarrow 0$,

$$
\mathbb{E}\left[T_{\omega_{1}}^{\varepsilon}(Z) \cdots T_{\omega_{n}}^{\varepsilon}(Z)\right] \rightarrow \mathbb{E}\left[\exp \left(\frac{i c_{H} B_{H}(Z)}{2} \sum_{j=1}^{n} \omega_{j}\right)\right]
$$

and then

$$
\begin{aligned}
& \mathbb{E}\left[a^{\varepsilon}\left(Z, s_{1}\right) \cdots a^{\varepsilon}\left(Z, s_{n}\right)\right] \rightarrow \frac{1}{(2 \pi)^{n}} \int \cdots \int e^{-i \sum_{j=1}^{n} s_{j} \omega_{j}} \widehat{f}\left(\omega_{1}\right) \cdots \widehat{f}\left(\omega_{n}\right) \\
& \times \mathbb{E}\left[\exp \left(\frac{i c_{H} B_{H}(Z)}{2} \sum_{j=1}^{n} \omega_{j}\right)\right] d \omega_{1} \cdots d \omega_{n} \\
&=\mathbb{E}\left[\frac{1}{(2 \pi)^{n}} \prod_{j=1}^{n} \int e^{-i\left(s_{j}-c_{H} B_{H}(Z) / 2\right) \omega} \widehat{f}(\omega) d \omega\right] \\
&=\mathbb{E}\left[\prod_{j=1}^{n} f\left(s_{j}-\frac{c_{H} B_{H}(Z)}{2}\right)\right] .
\end{aligned}
$$

The tightness proof is similar to the proof of Lemma 3.2 in [6], and the convergence of $a^{\varepsilon}(Z, s)$ follows. To conclude the proof of Theorem 1 it remains to prove the convergence of $b^{\varepsilon}(0, s)$. It is similar to the convergence of $a^{\varepsilon}(Z, s)$, up to substituting the application of (2.12) by that of (2.13).

Appendix A. Hermite polynomials. In this appendix we recall some results regarding Hermite polynomials that we use in this paper. We denote the Gaussian probability density of a random variable $X \sim \mathcal{N}(0,1)$ by

$$
g(x)=\frac{e^{-x^{2} / 2}}{\sqrt{2 \pi}}
$$

and we define for every $k \in \mathbb{N}$ the $k$ th Hermite polynomial by

$$
H_{k}(x)=(-1)^{k} \frac{g^{(k)}(x)}{g(x)} .
$$

The set of all Hermite polynomials $\left\{H_{k}, k=0,1,2, \ldots\right\}$ is an orthonormal base for the space $L^{2}(g(x) d x)=\left\{h: \mathbb{E}\left[|h(X)|^{2}\right]<\infty\right\}$. We denote by $J_{h}(k)$ (or $J(k)$ if there is no ambiguity) the projection coefficient of a function $h \in L^{2}(g(x) d x)$ on the subspace spanned by $H_{k}$, that is,

$$
J_{h}(k)=\mathbb{E}\left[H_{k}(X) h(X)\right]
$$

Then, we have the series representation

$$
h(x)=\sum_{k=0}^{\infty} \frac{J_{h}(k)}{k !} H_{k}(x),
$$

the convergence being in $L^{2}(g(x) d x)$. We can explicitly compute the second moments by

$$
\mathbb{E}\left[|h(X)|^{2}\right]=\sum_{k=0}^{\infty} \frac{\left|J_{h}(k)\right|^{2}}{k !} .
$$

Copyright $@$ ( ) by SIAM. Unauthorized reproduction of this article is prohibited. 
This formula is a direct consequence of the following relation that we use in this paper: for a centered two-dimensional Gaussian vector $\left(X_{1}, X_{2}\right)$ such that $\mathbb{E}\left[X_{1}^{2}\right]=\mathbb{E}\left[X_{2}^{2}\right]=1$ we have

$$
\mathbb{E}\left[H_{j}\left(X_{1}\right) H_{k}\left(X_{2}\right)\right]=\left\{\begin{array}{cll}
k ! \mathbb{E}\left[X_{1} X_{2}\right]^{k} & \text { if } & k=l \\
0 & \text { if } & k \neq l
\end{array}\right.
$$

\section{REFERENCES}

[1] M. Asch, W. Kohler, G. C. Papanicolaou, M. Postel, and B. White, Frequency content of randomly scattered signals, SIAM Rev., 33 (1991), pp. 519-625.

[2] P. Billingsley, Convergence of Probability Measures, Wiley, New York, 1968.

[3] L. Borcea, G. Papanicolaou, and C. Tsogka, Theory and applications of time reversal and interferometric imaging, Inverse Problems, 19 (2003), pp. S134-S164.

[4] L. Borcea, G. Papanicolaou, and C. Tsogka, Coherent interferometry in finely layered random media, Multiscale Model. Simul., 5 (2006), pp. 62-83.

[5] S. A. Bulgakov, V. V. Konotop, and L. VAzquez, Wave interaction with a random fat fractal: Dimension of the reflection coefficient, Waves in Random Media, 5 (1995), pp. 9-18.

[6] J. F. Clouet And J. P. Fouque, Spreading of a pulse travelling in random media, Ann. Appl. Probab., 4 (1994), pp. 1083-1097.

[7] L. Coutin, An introduction to (stochastic) calculus with respect to fractional Brownian motion, in Séminaire de Probabilités XL, Lecture Notes in Math. 1899, Springer, New York, 2007, pp. 3-65.

[8] L. COUtin AND Z. QIAN, Stochastic analysis, rough path analysis and fractional Brownian motions, Probab. Theory Related Fields, 122 (2002), pp. 108-140.

[9] S. Dolan, C. Bean, And B. Riollet, The broad-band fractal nature of heterogeneity in the upper crust from petrophysical logs, Geophys. J. Int., 132 (1998), pp. 489-507.

[10] A. Fannjiang And K. Solna, Scaling limits for laser beam propagation in atmospheric turbulence, Stoch. Dyn., 4 (2004), pp. 135-150.

[11] A. C. Fannjiang And K. Solna, Propagation and time reversal of wave beams in atmospheric turbulence, Multiscale Model. Simul., 3 (2005), pp. 522-558.

[12] A. Fannjiang and K. Solna, Superresolution and duality for time-reversal of waves in random media, Phys. Lett. A, 352 (2005), pp. 22-29.

[13] J. Feder, Fractals, Plenum Press, New York, 1988.

[14] J. P. Fouque, J. Garnier, G. Papanicolaou, and K. Solna, Wave Propagation and Time Reversal in Randomly Layered Media, Springer, New York, 2007.

[15] S. Frey, W. Geurts, And L. Woste, Laser remote sensing for characterization of planetary boundary layer properties, in CLEO 2001, Technical Digest, Optical Society of America, Washington, DC, 2001, p. 494.

[16] A. E. GARGETt, The scaling of turbulence in the presence of stable stratification, J. Geophys. Res., 93 (1988), pp. 5021-5036.

[17] J. Garnier AND K. SøLna, Effective transport equations and enhanced backscattering in random waveguides, SIAM J. Appl. Math., 68 (2008), pp. 1574-1599.

[18] J. Garnier and K. Solna, Random backscattering in the parabolic scaling, J. Statist. Phys., 131 (2008), pp. 445-486.

[19] F. Herrmann, A Scaling Medium Representation, A Discussion on Well-Logs, Fractals and Waves, Ph.D. thesis, Department of Geophysics, Delft University of Technology, Delft, The Netherlands, 1997.

[20] T. A. Hewett, Modeling reservoir heterogeneities with fractals, in Proceedings of the 4th International Geostatistics Congress, Terra Abstracts 4, Suppl. 3, 9, 1992.

[21] V. V. Konotop, Z. Fei, And L. Vazquez, Wave interaction with a fractal layer, Phys. Rev. E, 48 (1993), pp. 4044-4048.

[22] H. J. Kushner, Approximation and Weak Convergence Methods for Random Processes, MIT Press, Cambridge, MA, 1994.

[23] M. Ledoux, T. Lyons, and Z. Qian, Lévy area of Wiener processes in Banach spaces, Ann. Probab., 30 (2002), pp. 546-578.

[24] A. LeJAY, An introduction to rough paths, in Séminaire de Probabilités XXXVII, Lecture Notes in Math., Springer-Verlag, New York, Berlin, 2003.

Copyright (c) by SIAM. Unauthorized reproduction of this article is prohibited. 
[25] P. Lewicki, R. Burridge, And M. V. De Hoop, Beyond effective medium theory: Pulse stabilization for multimode wave propagation in high-contrast layered media, SIAM J. Appl. Math., 56 (1996), pp. 256-276.

[26] P. Lewicki, R. Burridge, and G. Papanicolaou, Pulse stabilization in a strongly heterogeneous medium, Wave Motion, 20 (1994), pp. 177-195.

[27] T. Lyons, Differential equations driven by rough signals, Rev. Mat. Iberoamer., 14 (1998), pp. 215-310.

[28] T. Lyons, Differential equations driven by rough signals (I): An extension of an inequality of L. C. Young, Math. Res. Lett., 1 (1994), pp. 451-464.

[29] T. Lyons And Z. Qian, System Control and Rough Paths, Oxford Mathematical Monographs, Oxford University Press, London, 2002.

[30] R. MARTY, Théorème limite pour une équation différentielle à coefficient aléatoire à mémoire longue, C. R. Acad. Sci. Paris Ser. I, 338 (2004), pp. 167-170.

[31] R. MARTY, Asymptotic behavior of differential equations driven by periodic and random processes with slowly decaying correlations, ESAIM Probab. Statist., 9 (2005), pp. 165-184.

[32] R. F. O'Doherty and N. A. Anstey, Reflections on amplitudes, Geophys. Prospecting, 19 (1971), pp. 430-458.

[33] H. Omre, K. Solna, And H. TJelmeland, Simulation of random functions on large lattices, in Geostatistics, A. Soares, ed., Kluwer Academic Publishers, Dordrecht, The Netherlands, 1993, pp. 179-199.

[34] M. Pilkington and J. P. Todoeschuck, Stochastic inversion for scaling geology, Geophys. J. Int., 102 (1990), pp. 205-217.

[35] G. Samorodnitsky and M. S. Taqqu, Stable Non-Gaussian Random Processes, Chapman and Hall, London, 1994.

[36] C. Sidi And F. Dalaudier, Turbulence in the stratified atmosphere: Recent theoretical developments and experimental results, Adv. Space Res., 10 (1990), pp. 25-36.

[37] K. SøLnA, Acoustic pulse spreading in a random fractal, SIAM J. Appl. Math., 63 (2003), pp. $1764-1788$.

[38] K. Solna and G. Papanicolaou, Ray theory for a locally layered medium, Waves in Random Media, 10 (2000), pp. 151-198.

[39] X. Sun AND D. L. JAGGARD, Wave interaction with a generalized Cantor bar fractal multilayers, J. Appl. Phys., 70 (1991), pp. 2500-2507.

[40] M. S. TAQQU, Weak convergence to fractional Brownian motion and to the Rosenblatt process, Z. Wahrsch. Verw. Gebiete, 31 (1975), pp. 287-302.

[41] M. S. TAQQU, Convergence of integrated processes of arbitrary Hermite rank, Z. Wahrsch. Verw. Gebiete, 50 (1979), pp. 53-83.

[42] E. Tromeur, E. Garnier, P. Sagaut, and C. Basdevant, Large eddy simulations of aerooptical effects in a turbulent boundary layer, J. Turbulence, 4 (2003), pp. 1-22.

[43] D. Washburn, D. W. Banton, T. T. Brennan, W. P. Brown, R. R. Butts, S. C. Coy, R. H. Dueck, K. W. Koenig, B. S. Masson, P. H. Peterson, R. W. Praus, G. A. Tyler, B. P. Venet, and L. D. Weaver, Airborne Laser Extended Atmospheric Characterization Experiment ( $A B L E A C E$ ), technical report, Phillips Laboratory, Kirtland Air Force Base, Albuquerque, NM, 1996.

[44] J. Weiss And D. Marsan, Scale properties of sea ice deformation and fracturing, C. R. Phys., 5 (2004), pp. 736-751.

Copyright $@$ by SIAM. Unauthorized reproduction of this article is prohibited. 